\title{
A Systematic Review for Auditory Training Effect Based on Korean Database
}

\author{
Sihun Park ${ }^{1,2}$, Woojae Han ${ }^{1,2,3}$ \\ ${ }^{1}$ Laboratory of Hearing and Technology, ${ }^{2}$ Division of Speech Pathology and Audiology, ${ }^{3}$ Research Institute of Audiology and Speech Pathology, \\ College of Natural Sciences, Hallym University, Chuncheon, Korea
}

\author{
청능훈련 효과에 대한 한국어 데이터베이스 기반의 체계적인 문헌 고찰 \\ 박 시 훈,2 \\ 한림대학교 자연과학대학 HearT 실험실1 , 언어청각학부 ${ }^{2}$, 청각언어연구소 ${ }^{3}$
}

\begin{abstract}
Auditory training should be necessary to improve listeners' performance in auditory tasks in terms of active listening. Many clinicians, thus, believe that it is a potential intervention and an important component of the effective rehabilitation for hearing-impaired listeners. In the present paper, we reviewed articles of the auditory training based on the Korean database which accumulated over 20 years and scrutinized their training effects. Using key terms 'auditory training,' 'aural rehabilitation' from four Korean electronic databases, 258 articles were systematically found from 1998 to present. Among them, 21 articles met our inclusion criteria which followed a procedure of the Preferred Reporting Items for Systematic Reviews and Meta-Analyses flow diagram. Then, their study quality was evaluated using ten pre-defined scientific and intervention-specific measures. Unfortunately, meta-analysis technically failed to confirm due to heterogeneity among the sample data. The average scores of qualitative evaluations for 21 articles were $9.62( \pm 2.40)$, while showing a large difference in the quality. Only 7 out of 21 articles statistically supported that the hearing-impaired listeners had a significant improvement after conducting the auditory training. Regardless, most of the articles analyzed couldn't include systematic standards for the auditory training effect to be concluded comprehensively. Our findings demonstrate that published evidence for efficacy of the auditory training in the hearing-impaired listeners isn't robust and thus can't be reliably used to guide intervention at this time. When highly-qualitied evidence provides its efficacy for people with hearing loss in many following studies, it will suggest appropriate research direction on the auditory training program for the clinical application in Korea.
\end{abstract}

Key Words: Auditory training effect, Systematic review, Participants-Intervention-Control-Outcomes-Study design, Study quality.

Received: June 7, 2019 / Revised: June 23, 2019 / Accepted: June 23, 2019

Correspondence: Woojae Han, Laboratory of Hearing and Technology, Division of Speech Pathology and Audiology, \#8603 Natural Science Building, Hallym University, 1 Hallymdaehak-gil, Chuncheon 24252, Korea

Tel: +82-33-248-2216 / Fax: +82-33-256-3420 / E-mail: woojaehan@hallym.ac.kr

\section{INTRODUCTION}

\section{Background}

세계 보건기구(World Health Organization, WHO)에 따르 면, 전 세계적으로 약 4억 6천6백만 명의 사람들이 청력손실을 갖고 있다(WHO, 2019). 청력손실로 인하여 난청인들은 1차적 으로 의사소통의 어려움을 보고할 수 있고, 2차적으로는 고용 제한, 우울, 사회적 고립, 삶의 질 감소의 어려움이 발생하기 때

(c) This is an Open Access article distributed under the terms of the Creative Commons Attribution Non-Commercial License (https://creativecommons.org/licenses/by-nc/4.0) which permits unrestricted non-commercial use, distribution, and reproduction in any medium, provided the original work is properly cited.
문에(Davis et al., 2007) 전문가의 관리가 필수적이다.

난청의 치료와 재활 중 가장 대표적인 방법은 보청기의 처방 이다. 그러나 청각학의 선진국으로 알려진 미국에서도 난청 환자 들 중 30.2\%만이 보청기를 소유하고 있으며(Abrams \& Kihm, 2015), 영국 또한 난청 환자 중 약 20\%만이 보청기를 소유하고 있다(Kochkin, 2009). 게다가 보청기를 소유한 환자들 중 약 15 30\%는 지속적으로 보청기를 착용하고 있지 않다(Kochkin, 2009). 많은 난청인들이 보청기를 받아들이지 못하거나 꺼 려하는 주된 이유는 보청기 착용 후에도 여전히 소음 속 상황 에서 말소리를 이해하기 어렵기 때문이다(Banh et al., 2012). 이는 재활에 대한 관점에서 보청기의 착용만으로는 난청인의 
의사소통의 불편함을 해소하기에 한계가 있음을 시사한다. 따 라서 많은 임상가들과 연구자들은 보청기를 포함한 다양한 청 각 보조기기의 정확한 처방과 더불어 체계적인 청능훈련이 수 반되어야 함을 주장하고 있다.

국내에서도 청각장애의 조기 발견 및 재활, 보청기 및 인공와 우 등의 청각 보조기기의 기술적 발전으로 고심도 난청인도 정 상적인 언어 습득 및 언어 발달의 가능성이 높아짐에 따라 청 능훈련의 중요성 또한 관심이 높아지고 있다(Jang, 2007). 이에 따라 국내 실정 및 한국어의 특성을 고려한 다양한 청능훈련 도구의 개발이 활발히 이루어지고 있지만, 아직 개발된 도구의 효과 검증에 대한 파일럿 데이터만 다수 존재하고 임상에 직접 적으로 적용하여 구체적인 이득을 보고한 연구들은 매우 드물 다. 게다가 청능훈련을 시행하고 이에 대한 효과를 자료 기반으 로 종합적으로 분석한 문헌 또한 제한적이다. 따라서 본 종설에 서는 20년 넘게 축적된 한국어 데이터 기반의 청각재활에 관한 체계적 문헌 검색을 통해 난청인의 청능훈련의 효과에 대해 확 인하고 이를 바탕으로 향후 청능훈련 프로그램 개발과 적용에 대한 연구 방향을 제언하고자 한다.

\section{Research aims}

체계적 문헌 고찰이란 주어진 연구 문제에 답하기 위해 사전 에 지정된 적격성 기준들을 충족하는 모든 경험적인 증거를 확 인하고 평가하며 종합하는 과정이다(Berman \& Parker, 2002). 이에 본 종설에서는 난청인들을 위한 효과적인 청능훈련에 대 해 체계적 문헌 고찰을 목표로 다음의 네 가지 절차에 따라 분 석하고 논의하였다. 첫째, 1998년부터 2019년 4월까지 출간된 청능재활과 청능훈련에 대한 연구 동향을 검색하고 분석한다. 둘째, 청능훈련의 일반적인 특성과의 연관성, 훈련의 효과에 대 하여 보고된 문헌들을 중심으로 세부적으로 분석한다. 셋째, 분석 논문들의 청능훈련을 위한 도구와 그에 대한 구성 항목 들을 면밀히 조사한다. 넷째, 향후 난청인을 대상으로 한 청능 훈련 프로그램 개발과 적용에 대한 기초 자료를 제공한다.

\section{MATERIALS AND METHODS}

\section{Methods of data extraction}

데이터 추출, 데이터 분석, 데이터 포함 및 제외 기준의 방법 은 체계적 문헌 고찰의 과정 내에서 사전에 지정되고 문서화하 였다. 즉, Participants, Intervention, Control, Outcomes, Study design (PICOS) 기준을 적용하여(Henshaw \& Ferguson, 2013) 문헌 분류를 진행하였으며(Table 1), 기준에 부합하지 않는 문 헌은 제외하였다. 이러한 기준의 적용은 자료 수집 전 명확하게 정의되어 검토 과정의 투명성을 제공할 수 있다(Henshaw \& Ferguson, 2013). 또한 체계적 문헌 고찰 과정의 세부 사항은 Moher et al.(2009)의 'Preferred Reporting Items for Systematic Reviews and Meta-Analyses 2009 Flow Diagram’을 참 고하여 작성하였다.

\section{Study identification}

본 연구는 국내 보장구의 처방과 상관없이 난청인들을 대상 으로 시행한 청능훈련과 관련된 연구만을 선정하고 수집하였 다. 구체적으로 문헌 선정을 위해 국내 4 개의 대표적 전자 데이 터 베이스인 '한국학술정보시스템(KISS)', '국회전자도서관', '구 글 학술검색' '네이버 학술정보'에서 참고 문헌들을 검색하였다. 검색어는 관련 문헌에서 표현하는 용어를 통해 선정하였다. 예 를 들어 사용된 검색어는 '청능훈련/재활' '청각훈련/재활', '보 청기 훈련/재활', '청각보조기기 재활', '인공와우 재활/훈련'을 적절히 조합하여 적용하였다.

문헌들의 출간 기간은 전 세계적으로 청능훈련에 대한 구체 적인 기반을 구축하고 제시한 Foundations of Aural Rehabilitation (Tye-Murray, 1998) 서적의 출간연도를 기준으로 문헌 의 출판 연도를 1998년부터 2019년 4월까지로 제한하였으며, 본 연구의 목적에 맞게 일반적인 인식 수준과 환경이 문화적 차이에 의해 다를 수 있는 국외 거주자를 대상으로 한 국외학 술지 및 국외서적은 배제하였다. 본 문헌 고찰에서 적용한 기준 들은 Table 2에 정리하였다.

Table 1. PICOS criteria for inclusion

\begin{tabular}{ll}
\hline \multicolumn{1}{c}{ PICOS } & \multicolumn{1}{c}{ Specific information } \\
\hline Participants & Physically hearing handicapped person \\
Intervention & Auditory training \\
Control & Comparison with a control group or repeated measures (pre- and post-training comparison) \\
Outcomes & $1+$ outcome measure(s) related to speech intelligibility, cognition or communication (either behavioral measures \\
& or self-reported outcomes) \\
Study design & Randomized controlled trials, non-randomized controlled trials, cohort studies (with a control comparison), and \\
& repeated measures (pre- and post-training comparisons) \\
\hline
\end{tabular}

PICOS: participants, intervention, control, outcomes, study design 
Table 2. Selection criteria for papers mentioned auditory training effect

\begin{tabular}{|c|c|c|}
\hline Type & Inclusion & Exclusion \\
\hline Paper publication year & 1998-2019 April & Before 1998, after 2019 April \\
\hline Types of literature & Domestic journals & Foreign journals, books \\
\hline Study design & $\begin{array}{l}\text { Cross-sectional study } \\
\text { Experimental studies (experimental design, non experimental } \\
\text { design cohort study) }\end{array}$ & $\begin{array}{l}\text { Meta-analysis and systematic analysis } \\
\text { essay (article) }\end{array}$ \\
\hline Contents & Auditory training, auditory rehabilitation effect & $\begin{array}{l}\text { Explanation of the rehabilitation method, } \\
\text { explanation of auditory training }\end{array}$ \\
\hline
\end{tabular}

Figure 1. Flow diagram of the study identification, eligibility, and inclusion process. PICOS: Participants, intervention, control, outcomes, study design, OCMs: outcomes.

\section{Screening}

4개의 전자 데이터 베이스 검색 엔진의 결과를 통하여 총 418개의 문헌이 관련 논문으로 검색되었다. 2019년 4월까지의 자료를 검색하기 위해 2019년 5월에도 추가 자료를 검색하였지 만, 추가적인 자료를 검색할 수 없었다(Identification level; Figure 1).

스크린 과정에서 중복 문헌들 $(\mathrm{n}=160)$ 을 제거한 후 잠재적 으로 관련이 있는 258 개의 문헌을 추출하였다. 258 건의 문헌들 의 초록을 PICOS 기준에 의거하여 2 명의 저자가 독립적으로 평가하였다. 즉, 초록에 대한 내용이 본 종설 논문의 주제와 무 관한 경우 해당 문헌을 제외하거나 초록의 내용이 PICOS 기준 에 충족되지 못한 213건의 문헌을 제외하여 45건의 문헌이 남 았다(Screening level; Figure 1). 45건의 문헌은 초록이 PICOS 기준에 충족되었거나 혹은 초록의 정보만으로는 판단을 내리 기에 충분하지 못한 내용을 포함하였다.

\section{Eligibility}

초록 검토 이후 정리된 45개 문헌의 전체 내용을 검토한 결 과 다양한 이유로 PICOS 기준을 충족시키지 못한 24개의 문
헌을 배제하였다(Eligibility level; Figure 1). 더불어 동일한 연 구 대상자의 결과에서 여러 개의 출판물이 발생한 경우 체계적 문헌 고찰의 Centre for Reviews and Dissemination(2008)에 근거하여 첫 번째 출판물만을 포함하였고 결과적으로 총 21 개 의 문헌만을 체계적 문헌 고찰에 포함하였다(Included level; Figure 1).

\section{Data extraction and data synthesis}

추출된 데이터는 데이터 추출 및 질적 평가 양식 내에서 사 전 지정되었으며, 두 명의 연구자가 시행해보고 필요에 따라 수 정을 진행하였다. 최종 연구 데이터 추출은 두 명의 연구자가 독립적으로 수행하여 내용을 포함하였다. 즉, 연구설계, 연구 대상자, 훈련에 사용된 자극음, 훈련 기간, 결과에 대한 측정, 주요 결과, 훈련 이후 향상된 능력의 지속성 확인을 포함하여 진행하였다. 추출된 자료에 대해 두 명의 연구자의 합의가 이루 어지지 않는 경우 합의에 도달할 때까지 문헌을 공동으로 재검 토하였다. 


\section{Study quality and potential sources of study bias}

과학적 연구의 질적 평가와 잠재적 연구의 편향됨(bias)은 무 작위 추출(randomization), 통제화(controls), 표본 수 산정 (sample size and power calculation), 맹검법(blinding), 결과 측정 보고(outcome measure reporting) 총 5 가지의 독립적인 방법으로 평가되었고, 본 논문에서는 5 가지 측정 방법을 추가 하여 진행하였다. 추가적인 5 가지 방법은 연구설계의 과정에서 훈련, 재활의 질적 타당성을 확보하기에 매우 구체적인 방법으 로써(Henshaw \& Ferguson, 2013), generalization of learning to functional benefits in real-world listening (outcome selection), training feedback, ecological validity, compliance with training protocols, and long term follow up of improvements였다. 10 가지 항목의 질적 평가를 분류를 통해 추출한 문 헌을 대상으로 실시하였다. 측정 점수가 낮으면 정보가 적은 것 을 의미하며, 결과적으로 연구의 높은 편향성을 포함할 가능성 이 높다. 질적 평가의 각 항목당 점수는 0 2의 점수로 구성되 어 있다. 0 점은 잘못되었거나 판단을 할 수 있는 정보가 없음을 나타내며, 1 점은 적은 정보를 포함하고 있거나 구체적인 정보가 없음을 의미하고, 2점은 구체적인 정보의 적절한 사용 및 보고 를 나타낸다.

각 문헌의 개별 척도 점수를 합산하여, levels of evidence를 평 가하였다. Levels of evidence는 2004 Grading of Recommendations Assessment, Development and Evaluation을 참고하 여 작성되었다. 이는 점수가 높을수록 훈련 효과 추정에 대한 신뢰성 있는 결과를 보여준다. 합산한 점수를 바탕으로 0 5의 점수는 매우 낮음, 6 10의 점수는 낮음, 11 15의 점수는 보통, 16 20의 점수는 높음의 수준을 보여준다. 낮은 수준의 levels of evidence를 보이는 문헌은 동일한 방법론적 접근의 반복적 인 연구에서 결과가 다양하게 나타날 수 있는 가능성이 높다. 반면, 높은 수준의 levels of evidence를 보이는 문헌은 동일한 방법론적 반복에도 유효한 결과를 보여주었다(Table 3).

연구 대상자(청력손실의 정도, 나이, 보청기 사용, 인공와우 사용)의 차이, 훈련에 사용된 자극음, 훈련 과정 및 결과 측정 의 측면에서 연구 간의 이질성(heterogeneity)으로 인해 비교
가능한 연구 결과의 메타 분석을 실시할 수 없었다. 따라서 연 구 결과 및 연구의 질적 평가는 결과의 해석과 체계적 문헌 고 찰에 포함된 21 개 문헌의 결과 차이를 조사하기 위해 구조적으 로 기술하였다.

\section{RESULTS}

\section{Study characteristics}

21 개의 문헌에서 추출한 데이터는 PICOS 기준에서 제시하 였다. 21 개의 문헌에서 연구의 질적 평가를 진행하기 위해 추출 한 데이터를 분석하고 요약하였다(Table 4).

\section{Participants}

연구 대상자는 청각 보조기기를 사용하지 않는 난청인(Yoon et al., 2016)을 제외하고 대부분 보청기 혹은 인공와우를 착용 하거나 보청기와 인공와우를 함께 사용하였다. 나이는 9 개월 (Yoon et al., 2016)부터 87세(Kim \& Lee, 2010)까지 다양하였 다. 연구 대상자 수는 1명(Cho \& Lee, 2010; Cho et al., 2013, 2018; Hong, 2013; Kim \& Lee, 2018; Lee \& Cho, 2011; Lee \& Seo, 2015; Yoon et al., 2016; Yu et al., 2014)에서 최대 24명 (Kim \& Lee, 2010)이었다.

\section{Intervention}

훈련 자극음, 훈련 빈도, 회차별 훈련 시간 및 훈련 기간은 연구마다 다양했다. 청능훈련에 사용한 훈련 자극음은 Ling 6 음(Cho \& Lee, 2010; Hong, 2013; Lee \& Cho, 2011; Lee \& Seo, 2015; Park et al., 2003), 자음과 모음 및 단음절 단어와 다음절 단어(Hong, 2013; Kim \& Lee, 2010; Lee \& Cho, 2011; Lee et al., 2016; Park \& Lee, 2005; Park et al., 2003), 악기소리 및 음악(Cho \& Lee, 2010; Choi et al., 2017; Lee \& Cho, 2011; Park \& Lee, 2005), 주변 환경음 및 소음 속 환경 음(Hong, 2013; Kim \& Lee, 2017, 2018; Lee \& Seo, 2015; Lee et al., 2016), 의성어 및 훈련 대상자에게 친숙한 어휘(Cho \& Lee, 2010; Hong, 2013; Kim \& Seok, 1998; Lee \& Cho,

Table 3. Levels of evidence by study quality score

\begin{tabular}{|c|c|c|}
\hline $\begin{array}{l}\text { Scores of } \\
\text { study quality }\end{array}$ & $\begin{array}{l}\text { Levels of } \\
\text { evidence }\end{array}$ & Confidence in estimation of effect \\
\hline $0-5$ & Very low & The estimation of effect is uncertain \\
\hline $6-10$ & Low & $\begin{array}{l}\text { Further evidence is very likely to impact on our confidence in the estimation of effect and are likely to } \\
\text { changethe estimate }\end{array}$ \\
\hline $11-15$ & Moderate & $\begin{array}{l}\text { Further evidence is likely to impact on our confidence in the estimation of effect and may change } \\
\text { the estimate }\end{array}$ \\
\hline $16-20$ & High & Further evidence is very unlikely to change our confidence in the estimation of effect \\
\hline
\end{tabular}




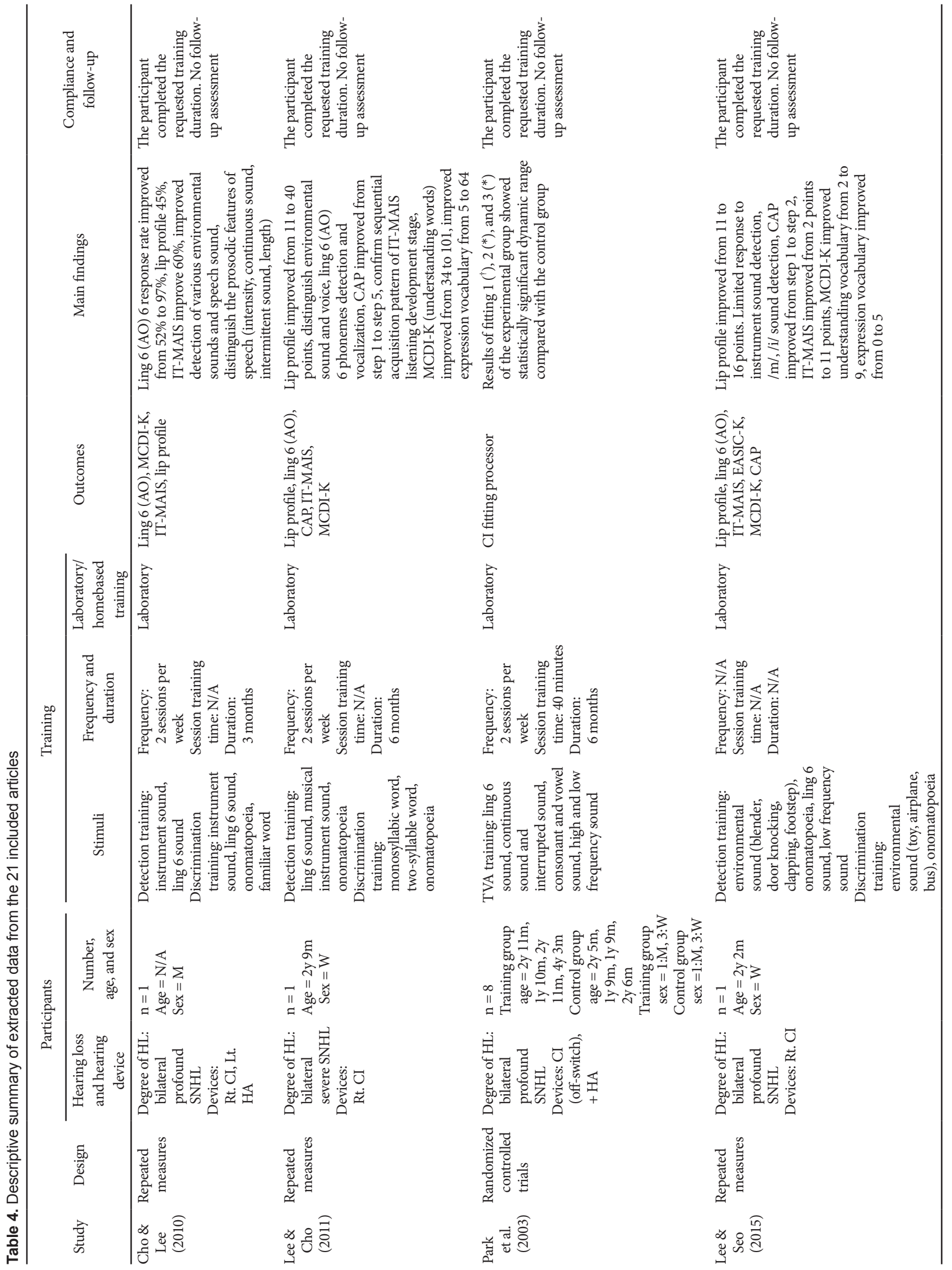




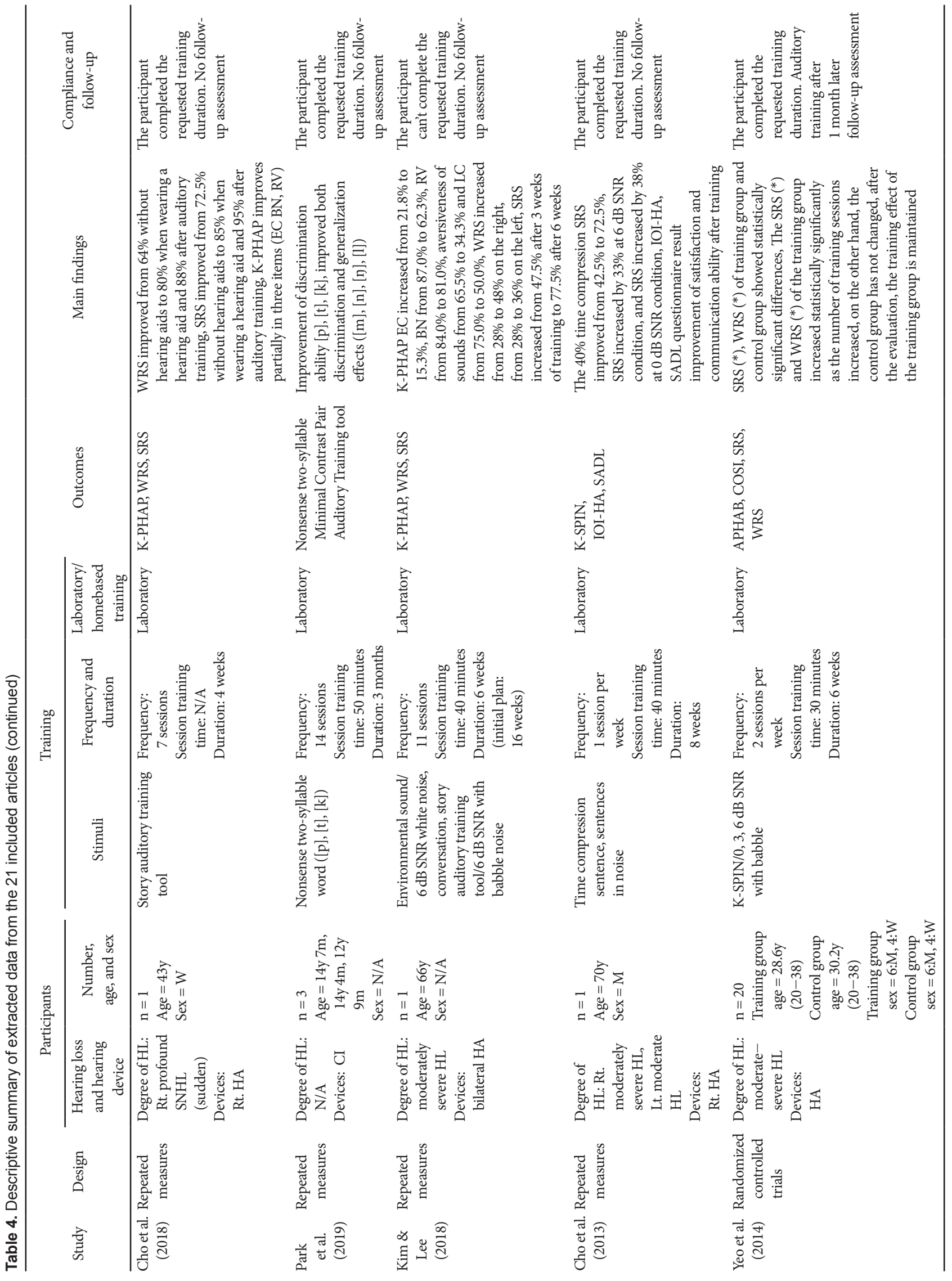




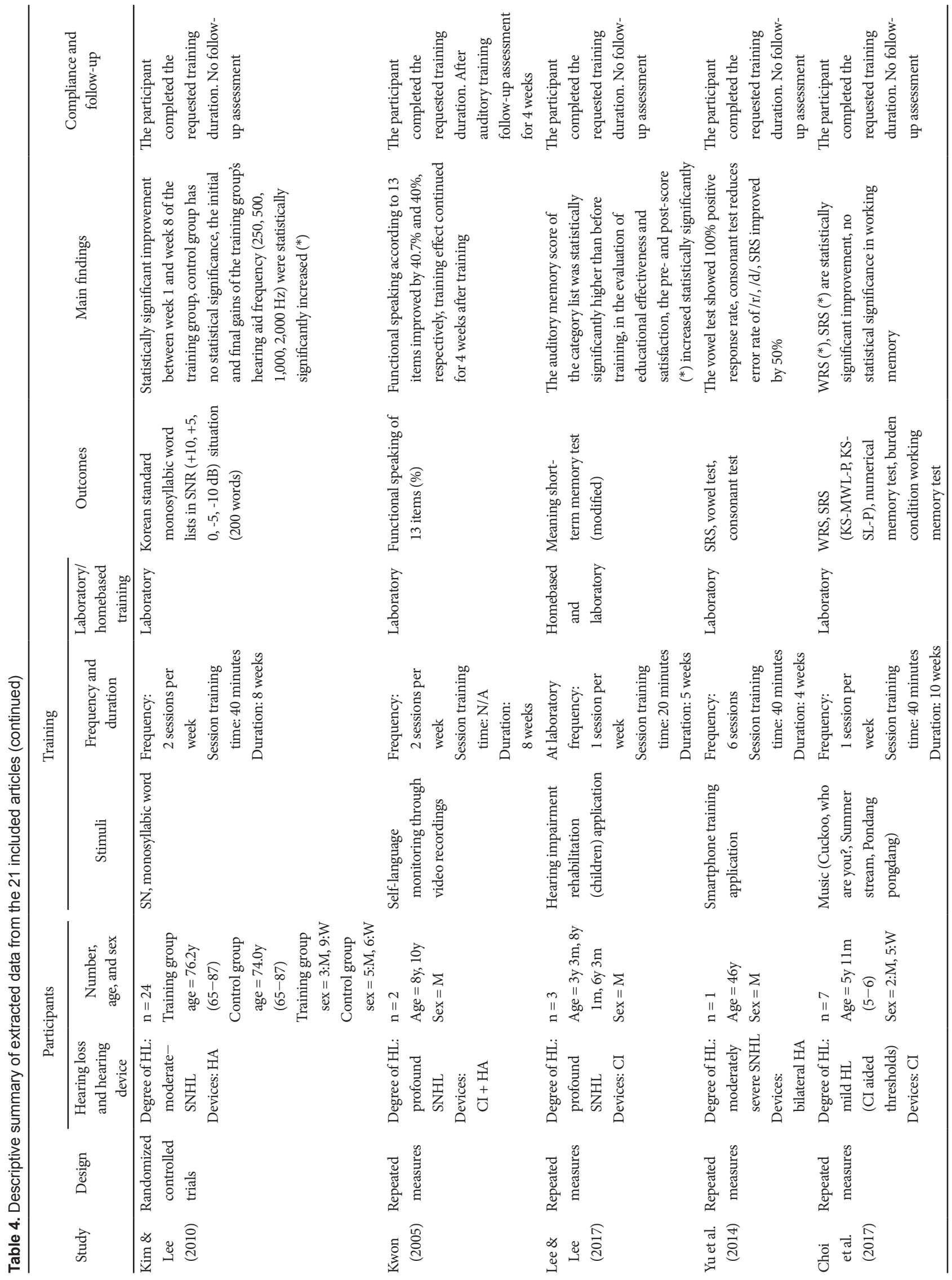




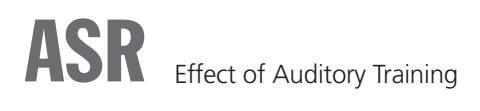

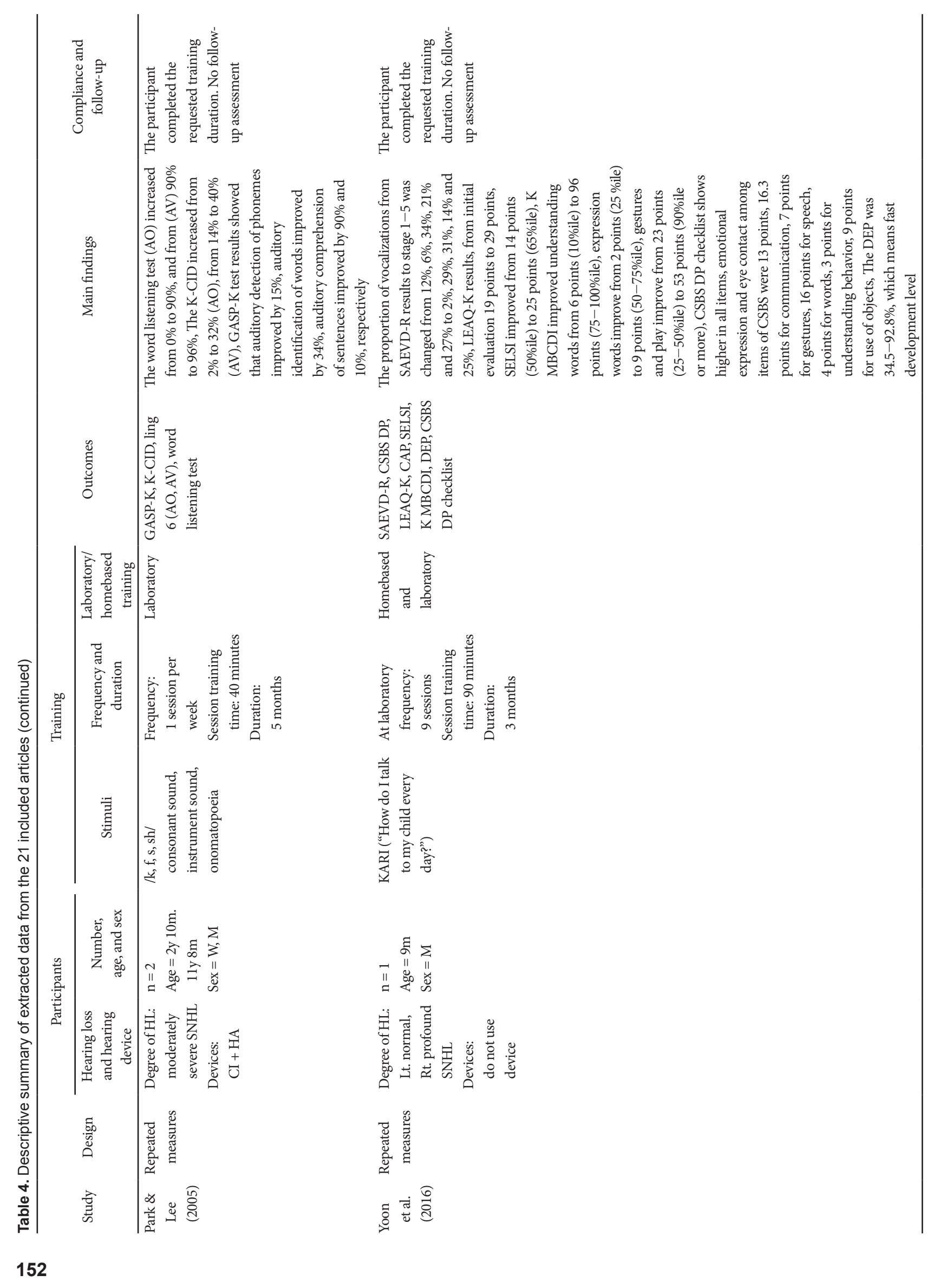




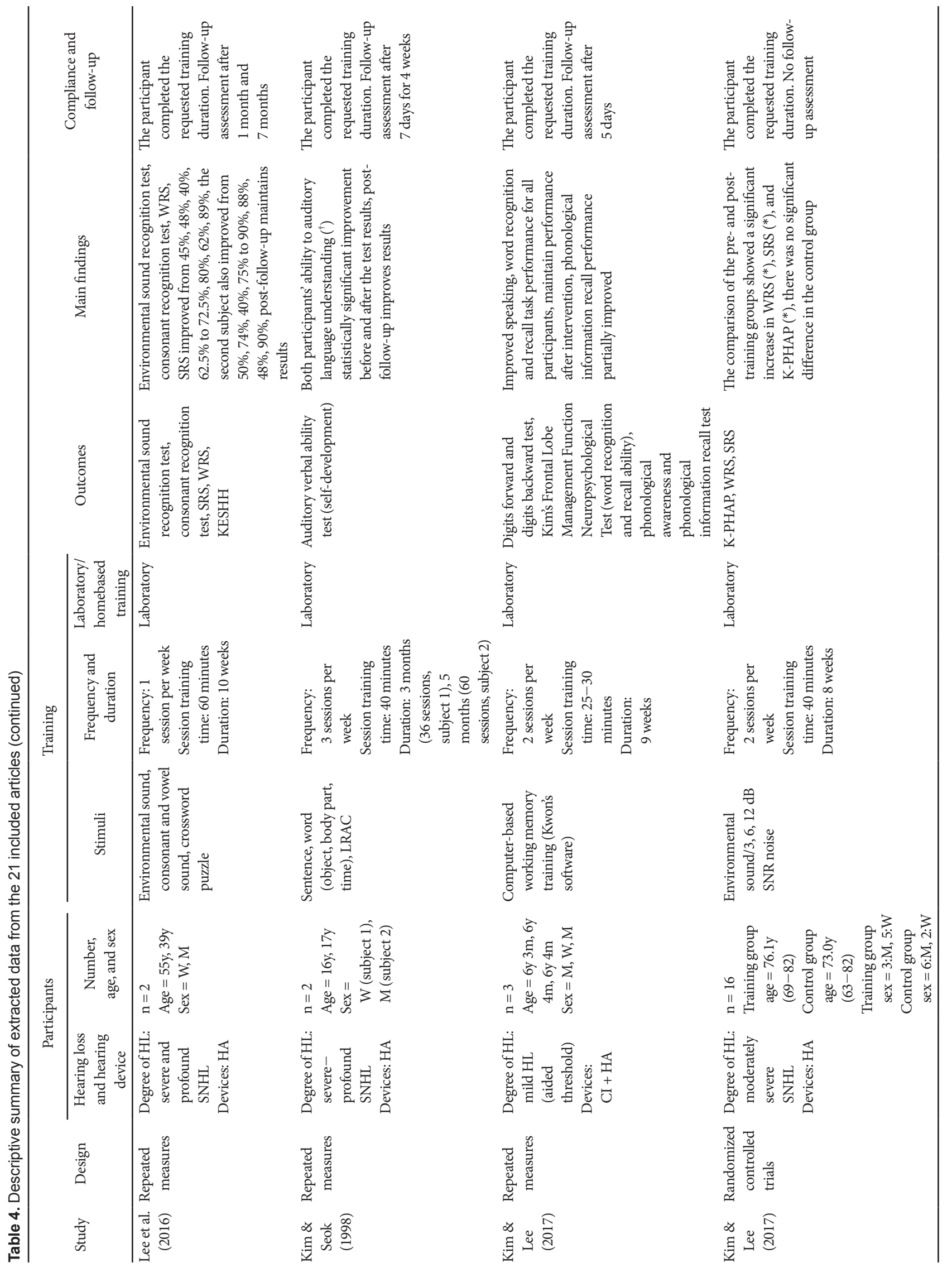




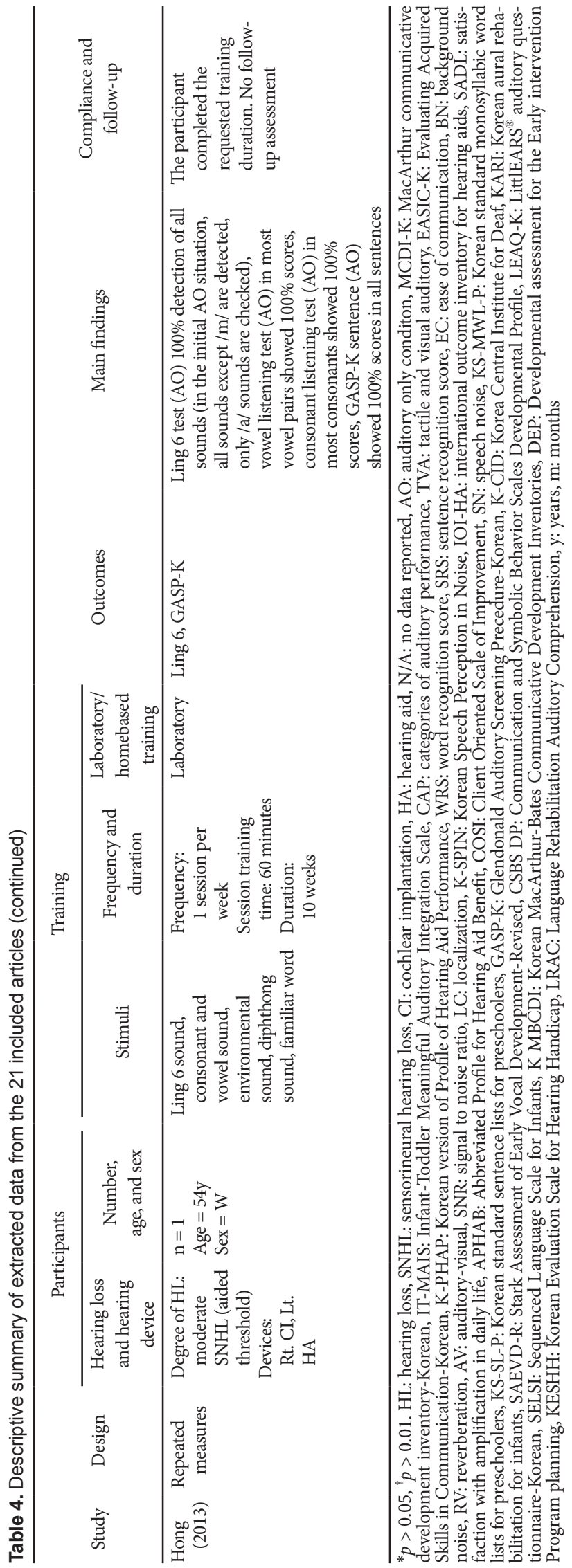

2011; Lee \& Seo, 2015; Park \& Lee, 2005), 단속음과 연속음 (Park et al., 2003), 문장, 시간 압축 문장, 소음하 문장 음원 및 소음하 대화(Cho et al., 2013; Kim \& Lee, 2010, 2018; Kim \& Seok, 1998; Yeo et al., 2014), 고음 및 저음(Lee \& Seo, 2015; Park et al., 2003), 무의미 이음절 단어(Park et al., 2019), 이 외에 스마트폰 어플리케이션을 활용한 청능훈련 음원(Lee \& Lee, 2017; Yu et al., 2014), 컴퓨터 기반 작업기억 훈련 음원 (Kim \& Lee, 2017), 이야기 청능훈련 도구(Cho et al., 2018), 비디오 녹화 자료를 통한 본인의 언어(Kwon, 2005), Korean Aural Rehabilitation for Infants의 재활도구(Yoon et al., 2016), 가로세로 단어판 도구(Lee et al., 2016)로 다양했다.

1주당 훈련 회기는 1회(Cho et al., 2013; Choi et al., 2017; Hong, 2013; Lee \& Lee, 2017; Lee et al., 2016; Park \& Lee, 2005)에서 최대 3회(Kim \& Seok, 1998)였다. 훈련 시간은 회 기당 20분(Lee \& Lee, 2017)에서 최대 90분(Yoon et al., 2016) 이었다. 훈련 기간은 4주(Cho et al., 2018; Yu et al., 2014)에서 최대 6개월(Lee \& Cho, 2011; Park et al., 2003)이었다. 모든 문헌이 실험실 기반의 훈련이었으나, 가정과 실험실 기반 훈련 을 통합하여 진행한 연구(Lee \& Lee, 2017; Yoon et al., 2016) 도 있었다.

\section{Controls}

엄격히 말해 무작위 대조군 연구(randomized controlled trials, RCTs)의 체계적 고찰과 메타 분석은 근거 중심의 기준 (evidence-based guidelines)의 등급 체계에 있어서 매우 높은 수준의 근거로 여겨진다(Berman \& Parker, 2002). 그러나 본 체계적 문헌 고찰에서는 무작위 대조군 연구만을 포함할 경우 난청인에게 적용한 청능훈련의 효과를 포함한 대부분의 문헌 은 해당되지 않기에 다른 기법이 고려되었다. 즉, 통제집단과 훈 련 집단을 비교하거나, 동일 대상자의 훈련 전과 훈련 후를 직 접 비교한 연구만이 본 체계적 문헌 고찰에 포함되었고, 반복 측정 연구설계(repeated measures design)가 가장 일반적인 연 구설계였다.

\section{Outcome measures}

모든 연구의 결과 측정 방법을 공통화하는 것은 불가능하였 다. 결과 측정 방법의 예로써, Ling 6 청각자극단서 상황(auditory only) 혹은 청시각단서 상황(auditory-visual) (Cho \& Lee, 2010; Hong, 2013; Lee \& Cho, 2011; Lee \& Seo, 2015; Park $\&$ Lee, 2005), 단어인지도검사 혹은 문장인지도검사(Cho et al., 2018; Choi et al., 2017; Kim \& Lee, 2017, 2018; Lee et al., 2016; Yeo et al., 2014; Yu et al., 2014), MacArthur communicative development inventory-Korean (Cho \& Lee, 2010; 
Lee \& Cho, 2011; Lee \& Seo, 2015), Infant-Toddler Meaningful Auditory Integration Scale (Cho \& Lee, 2010; Lee \& Cho, 2011; Lee \& Seo, 2015), Lip profile (Lee \& Cho, 2011; Lee \& Seo, 2015), categories of auditory performance (Lee \& Cho, 2011; Lee \& Seo, 2015; Yoon et al., 2016), Korean version of Profile of Hearing Aid Performance (K-PHAP) 혹 은 Abbreviated Profile for Hearing Aid Benefit (APHAB) (Cho et al., 2018; Kim \& Lee, 2010, 2017; Yeo et al., 2014), Evaluating Acquired Skills in Communication-Korean (Lee \& Seo, 2015), Korean Speech Perception in Noise 혹은 소음 속 단음절어 평가(Cho et al., 2013; Kim \& Lee, 2010), 자음 혹은 모음 검사(Lee et al., 2016; Yu et al., 2014), international outcome inventory for hearing aids (Cho et al., 2013), satisfaction with amplification in daily life (Cho et al., 2013), Client Oriented Scale of Improvement (Yeo et al., 2014), Glendonald Auditory Screening Precedure-Korean (Hong, 2013; Park \& Lee, 2005), Korea Central Institute for Deaf (Park \& Lee, 2005), Stark Assessment of Early Vocal Development-Revised, Communication and Symbolic Behavior Scales Developmental Profile (CSBS DP), LittlEARS ${ }^{\circledR}$ auditory questionnaire-Korean, Sequenced Language Scale for Infants, Korean MacArthur-Bates Communicative Development Inventories, Developmental assessment for the Early intervention Program planning, CSBS DP checklist (Yoon et al., 2016), Korean Evaluation Scale for Hearing Handicap (Lee et al., 2016)이 있었고 자체 개발 도구 또는 기 존의 검사를 수정한 결과 측정 도구로는 환경음 인지도 평가 (Lee et al., 2016), 청각적 언어이해능력 검사지(Kim \& Seok, 1998), 숫자 바로 따라 말하기, 숫자 거꾸로 따라하기 과제, Kim's 전두엽 관리 기능 신경심리검사, 음운 인식과 음운적 정 보 회상 검사(Kim \& Lee, 2017), 무의미 이음절 최소 대조짝 도구(Park et al., 2003), 13항목의 기능적 말하기 정반응(Kwon, 2005), 의미 단기기억검사(Lee \& Lee, 2017), 숫자 회상기억검 사, 부담 조건 작업기억검사(Choi et al., 2017), 낱말듣기검사 (Park \& Lee, 2005)가 있었다. 이 외에 cochlear implantation fitting software를 이용한 C-level, T-level 그리고 역동 범위 를 결과 측정 방법(Park et al., 2019)으로 사용한 연구도 있었다.

\section{Study designs}

질적 평가 대상의 21 개 문헌 연구 결과에서 대부분의 연구 가 반복측정 연구설계(repeated measures design)였으며, 그 중 4개의 문헌(Kim \& Lee, 2010, 2017; Park et al., 2003; Yeo et al., 2014)이 무작위 대조군 연구(RCTs)에 해당하였다.

\section{On-task learning and generalization of on-task learning}

온-태스크 학습은 일반적으로 직접 훈련받은 과제 수행 또 는 훈련 자극음에 대한 수행력 향상으로 정의할 수 있다(Henshaw \& Ferguson, 2013). 모든 문헌에서 훈련받은 과제 혹은 훈련 자극음에 대한 수행력이 향상되었다. 그러나 대부분의 문 헌이 통계 분석을 실시하지 않아 청능훈련 후의 수행력 향상이 통계적으로 유의한지에 대해서는 확인할 수 없었다. 통계 분석 을 실시한 문헌(Choi et al., 2017; Kim \& Lee, 2010, 2017; Kim \& Seok, 1998; Lee \& Lee, 2017; Park et al., 2003; Yeo et al., 2014)은 총 7개였다.

학습의 일반화는 직접적으로 훈련되지 않은 과제 수행 또는 훈련 자극음에 대한 수행력 향상으로 정의된다. 학습의 일반화 를 측정한 결과는 어음 명료도, 어음 인지 및 자가보고된 의사 소통의 향상(Henshaw \& Ferguson, 2013)의 세 가지 하위 목 록으로 나뉘어져 있다. 통계 분석을 실시한 7 개의 문헌을 분석 한 결과 기준에 의해 일반화를 측정할 수 있었던 문헌은 총 6 개였다. Park et al.(2003)의 다감각 청능훈련은 청각적 자극과 함께 다른 감각 자극을 동시에 제시하는 방식이었으며, 주된 훈 련의 목표는 소리의 탐지였다. 결과 측정은 인공와우 매핑 프로 그램을 사용하여 T-level, C-level, 역동 범위를 측정하며 훈련 집단과 통제집단과의 유의미한 결과를 보여주었다. 음의 탐지 훈련의 결과로 인한 향상을 보여주었고 이 외에 일반화를 도출 할 다른 수행력 향상 결과를 제시하지 않아 기준에 의한 일반 화를 측정할 수 없었다. Yeo et al.(2014)은 문장과 단어를 이용 하여 소음하 청능훈련을 실시하였다. 결과적으로 소음하 단어 인지도검사(word recognition score, WRS)와 문장인지도검사 (sentence recognition score, SRS)에서 통제집단과 훈련집단의 유의미한 결과를 보여주었다. Kim \& Lee(2010)는 단어를 이용 하여 소음하 청능훈련을 실시하였다. 결과적으로 소음하 WRS로 평가하였다. 훈련그룹의 1주 차와 8주 차간 통계적 유 의미한 향상을 확인할 수 있었고, 훈련그룹의 보청기 주파수별 $(250,500,1,000,2,000 \mathrm{~Hz})$ 최종 이득이 증가하여 통계적으로 유의미한 결과를 보여주었다. Kim \& Seok(1998)은 청각적 언 어 재활을 Language Rehabilitation Auditory Comprehension을 통해 문장과 단어 위주로 진행하였다. 청각적 언어이해능 력 검사지를 통해 검사를 진행하였고 훈련 전과 후에 통계적으 로 유의미한 향상을 보였으며, 문헌 내에서 일반화 효과가 있었 음을 통계적으로 직접 제시하였다. Kim \& Lee(2017)는 소음하 상황에서 환경음을 듣고 과제를 수행하는 청능훈련을 실시하 였다. 평가는 훈련하지 않았던 WRS, SRS, K-PHAP를 검사로 실시하였고 훈련그룹의 훈련 전과 훈련 후에 통계적 유의미한 향상을 보였다. Choi et al.(2017)은 아동들에게 익숙한 동요를 통한 청능훈련을 실시하였다. 결과 측정 방법으로 WRS와 


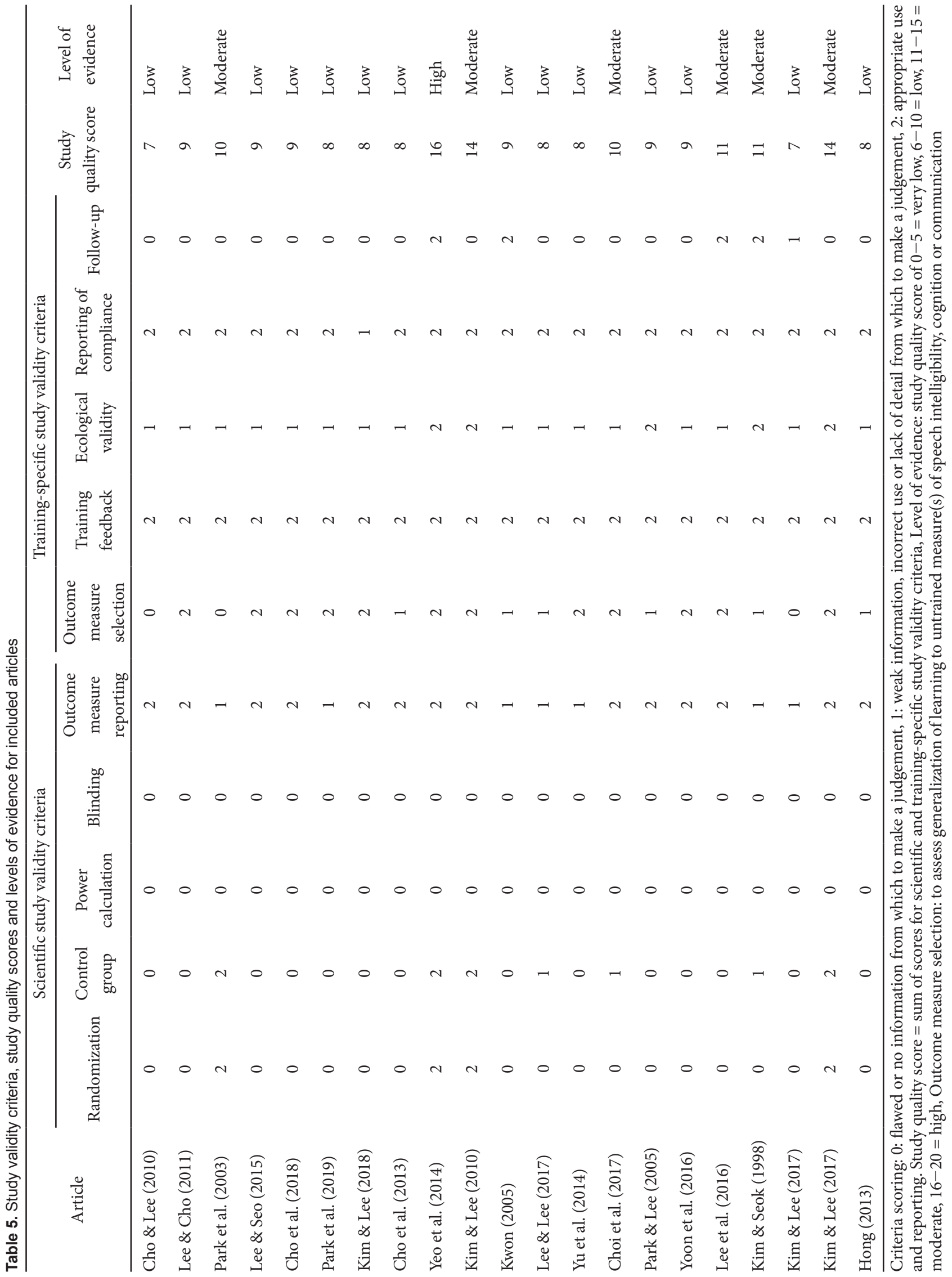


$\mathrm{SRS}$ 를 실시하였고 훈련 전과 후의 통계적 유의미한 상승을 보 고하였다. Lee \& Lee(2017)는 아동용 청각장애 재활훈련 어플 리케이션을 활용한 훈련을 실시하였다. 평가는 의미단기기억검 사를 수정하여 진행하였고 어플리케이션 청능훈련 효과에 대 한 자가보고를 포함하여 진행되었다. 훈련 전과 후의 통계적 유 의미한 향상을 보고하였다. 이 외에 직접적으로 일반화 효과에 대해 보고한 문헌은 1건(Park et al., 2019)이었다. Park et al.(2019)은 무의미 이음절 최소 대조짝을 이용하여 청능훈련 을 실시하였다. 훈련은 종성 파열음 [p], [t], [k]의 변별 능력 향 상을 주된 목표로 하였다. 결과적으로 종성 파열음 [p], [t], [k] 의 변별 능력이 모두 향상되었으며 더불어 비훈련 종성 [m], [n], [y], [1]의 변별 자질 일반화 효과 모두 향상되었다. 통계 분석을 실시하지 않았으나, 3 가지 기준에 의한 일반화를 측정할 수 있 었던 문헌으로는 어음 명료도(Kwon, 2005; Lee \& Cho, 2011; Lee \& Seo, 2015; Yoon et al., 2016; Yu et al., 2014), 어음 인지 (Cho et al., 2018; Choi et al., 2017; Hong, 2013; Kim \& Lee, 2018; Lee \& Cho, 2011; Lee \& Seo, 2015; Lee et al., 2016; Park \& Lee, 2005; Yoon et al., 2016; Yu et al., 2014), 자가보 고된 의사소통의 향상(Cho et al., 2013, 2018; Kim \& Lee, 2018; Lee \& Lee, 2017)이었다.

\section{Retention of learning}

학습 유지는 일반적으로 훈련 전 기초선 수행 수준으로부터 의 상당한 개선이 유지되는 경우 혹은, 사후 추적 관찰(followup assessment)에서 훈련 성과의 유의하지 않은 감소로 정의되 었다(Henshaw \& Ferguson, 2013). 사후 추적 관찰은 훈련 후 5일(Kim \& Lee, 2017)부터 최대 7개월(Lee et al., 2016)까지 진행되었다. 총 5 건 문헌에서 보고되었다. 사후 추적 관찰을 보 고한 5건의 문헌 중 4건(Kim \& Seok, 1998; Kwon, 2005; Lee et al., 2016; Yeo et al., 2014)의 문헌이 훈련 후 수행력이 유지 되거나 향상되었다. 1건(Kim \& Lee, 2017)의 문헌에서는 사후 추적 관찰에 대한 결과를 보고하지 않았다.

\section{Compliance with training}

훈련에 대한 규정 준수는 각 연구에서 요청된 훈련 기간을 완 료한 참가자의 비율로 정의된다(Henshaw \& Ferguson, 2013). 21개의 질적 평가 대상 문헌 모두 훈련에 대한 규정 준수 결과 를 보고하였으며, 20건의 문헌이 훈련에 대한 규정 준수를 $100 \%$ 완료하였다. 그중 1건의 문헌(Kim \& Lee, 2018)만이 초 기 요청되었던 훈련 기간을 완료하지 못하였다.

\section{Quality assessment}

연구의 질적 평가는 과학적 연구 타당도의 5 가지 측정법과
5 개의 교육 관련 연구 질적 평가 기준을 사용하여 평가되었으 며, 각 항목당 점수는 0 점에서 최대 2점까지였다. 즉, 연구의 질 적 평가는 10 개의 항목을 바탕으로 최대 20점 만점으로 계산 되었다. Table 5 는 21 건의 질적 평가 대상에 있는 문헌의 개별 연구 타당도 및 전체 연구의 질적 평가 결과이다. 21 건의 문헌 의 질적 평가를 진행한 결과 평균 $9.62( \pm 2.40)$ 점으로 전반적 으로 낮음(low) 수준이었다. 14건의 문헌이 낮음(low) 수준이 었으며, 중간(moderate) 수준의 문헌은 총 6건, 높음(high) 수준의 문헌은 총 1 건이었다.

\section{Scientific study quality}

과학적 연구 질적 평가 5 가지 항목에 대해 총점 10 점 중 가 장 높은 점수를 받은 문헌은 총 6점을 획득했다(Kim \& Lee, 2010, 2017; Yeo et al., 2014). 21건의 문헌 모두 연구 참가자 수 를 결정하는 데 필요한 객관적 통계지수를 확인할 수 없었다. 무작위 대조군 연구설계를 바탕으로 진행한 연구에서는 모든 문헌이 적절한 근거와 세부 사항을 바탕으로 진행하였다. 모든 문헌에서 훈련 결과에 대한 연구 대상자의 수행력을 보고하였 다. 자체 검사도구를 개발하거나 기존의 검사를 수정하여 진행 한 경우 검사도구에 대한 결과의 신뢰성이 떨어지므로 객관화 된 도구를 사용하였으며, 이에 대한 적절한 결과를 제시한 경우 에 2점을 받았다.

Training intervention-specific study quality

훈련 과정에서 모든 문헌이 성과 피드백을 사용한다고 보고 하였다. 또한 훈련에 대한 규정 준수 결과를 보고하였으며, 1 건 의 문헌(Kim \& Lee, 2018)만이 초기 요청되었던 훈련 기간을 완료하지 못하였다. 학습의 일반화는 3가지 기준에 의거하여 문 헌 내에서 확인할 수 있고 2가지 기준 이상의 향상된 수행력을 통해 일반화할 수 있는 경우 또는, 문헌 내에서 일반화에 대한 자체 보고가 있을 경우 2점을 부여하였다. 사후 추적 관리는 사 후 추적에 대한 내용이 문헌에 기술되어 있고, 사후 추적 결과 에 대한 정보가 있을 경우 2점을 부여하였다.

\section{CONCLUSIONS}

본 문헌 고찰의 주된 목적은 청각 보조기기 유무와 상관없 이 난청인을 위한 청능훈련의 효과를 체계적으로 검토하기 위 함이며, 부차적으로 향후 난청인을 대상으로 실시할 청능훈련 프로그램 개발과 적용에 대한 재활 효과적 측면에서 기초 자료 를 제공하기 위해 접근하였다.

일반적으로 체계적 문헌 고찰의 결과는 견고한 온-태스크 학습을 입증한다(Henshaw \& Ferguson, 2013). 본 고찰에서 
분석한 21건의 문헌에서 모두 훈련 과제를 통하여 연구 대상자 들은 하나 이상의 향상된 수행력을 보였다. 그러나 이러한 결과 는 검색된 문헌들의 정보만을 바탕으로 일반화하기에는 무리가 있었다. 첫째, 연구 대상자의 수가 대부분 10 명 이하(18/21)였 다. 둘째, 사례 보고의 문헌이 주를 이루었다. 셋째, 향상된 수 행력의 통계적 유의성에 대한 통계 분석이 몇몇 문헌(7/21)에서 만 실시되었다. 마지막으로, 전반적인 문헌의 정보 증거 수준이 강하지 못하였다. 이로써 본 체계적 문헌 고찰의 종합적인 결론 은 청능훈련은 난청인의 수행력 향상에 도움을 주는 것은 확인 이 가능했으나, 재활 방법에 따른 효과의 상대적인 크기를 확 인하기에는 현재까지 출간된 문헌들로는 어려움이 있었다.

더불어 본 체계적 문헌 고찰에서는 메타 분석을 진행하기에 다음과 같은 기술적 한계를 가졌다. 첫째, 20년 넘게 출간된 청 능훈련에 관한 논문들은 대부분이 사례 연구였다. 두 번째, 통계 분석을 실시한 문헌이 매우 제한적이었다. 세 번째, 연구 대상자 훈련 과정, 훈련 결과에 대한 이질성이 논문들 간 매우 높았다.

체계적 문헌 고찰과 더불어 무작위 대조군 연구(RCTs)는 등 급 체계에서 높은 증거 수준으로 여겨진다(Berman \& Parker, 2002). 현재로서는 증거 수준을 바탕으로 재활을 이끌 수 있는 결과를 제시하기에는 어려움이 따른다. 그러므로 앞으로 진행 될 청능훈련 및 청각재활 연구의 접근은 사례 연구보다는 문헌 의 증거 수준(evidence)이 높은 무작위 대조군 연구(RCTs) 기 반의 청능훈련 연구가 요구되며, 충분한 샘플 수를 바탕으로 연 구에 대한 통계 분석을 제시하여 청능훈련의 유의미성을 확인 하는 작업이 매우 절실하다. 높은 증거 수준의 연구와 다양한 청능훈련 연구에 대한 결과 측정의 표준화를 통해 난청 환자의 재활로 청능훈련의 효과를 적절하게 평가할 수 있는 근거 제공 의 필요성이 요구된다. 즉, 앞서 말한 높은 질적 수준의 연구를 종합해 메타 분석을 진행하여 효과적인 청능훈련에 대한 고찰 및 앞으로 한국인에 맞는 청능훈련 모델의 필요성이 강구된다.

중심 단어 : 청능훈련 효과·체계적 문헌 고찰·피코스(PICOS) · 연구의 질.

\section{Ethical Statement \\ N/A}

\section{Acknowledgments \\ N/A}

\section{Declaration of Conflicting Interests}

There are no conflict interests.

\section{Funding}

This work was supported by the Ministry of Education of the Republic of Korea and the National Research Foundation of Korea (NRF2018S1A3A2074932).

\section{Author Contributions}

W.H. designed the frame of the manuscript. S.P. worked for systematically searching the articles and analyzed the study quality. They wrote the manuscript and W.H. reviewed its final version as the corresponding author.

\section{ORCID iDs}

Sihun Park https://orcid.org/0000-0001-6085-2107

Woojae Han https://orcid.org/0000-0003-1623-9676

\section{REFERENCES}

Abrams, H. B. \& Kihm, J. (2015). An introduction to MarkeTrak IX: A new baseline for the hearing aid market. The Hearing Review, 22(6), 16.

Atkins, D., Best, D., Briss, P. A., Eccles, M., Falck-Ytter, Y., Flottorp, S., et al.; GRADE Working Group. (2004). Grading quality of evidence and strength of recommendations. BMJ: British Medical Journal, 328(7454), 1490.

Banh, J., Singh, G., \& Pichora-Fuller, M. K. (2012). Age affects responses on the speech, spatial, and qualities of hearing scale (SSQ) by adults with minimal audiometric loss. Journal of the American Academy of Audiology, 23(2), 81-91; quiz 139-140.

Berman, N. G. \& Parker, R. A. (2002). Meta-analysis: Neither quick nor easy. BMC Medical Research Methodology, 2, 10.

Centre for Reviews and Dissemination. (2008). Systematic Reviews: CRD's Guidance for Undertaking Reviews in Health Care. York: CRD, University of York

Cho, S. J. \& Lee, M. S. (2010). Aural rehabilitation after cochlear implantation in a child with keratitis-ichthyosis-deafness (KID) syndrome. $A u$ diology and Speech Research, 6(1), 93-97.

Cho, S., Oh, S. H., \& Bahng, J. (2018). A case study of auditory training for an adult with sudden sensorineural hearing loss. Audiology and Speech Research, 14(1), 59-64.

Cho, Y. Y., Bahng, J., \& Lee, J. H. (2013). Case study of auditory training for an elderly hearing aid user. Audiology and Speech Research, 9(2), 190194.

Choi, W. J., Oh, S. H., \& Bahng, J. (2017). Efficacy of music training on speech recognition and working memory in children wearing cochlear implants. Audiology and Speech Research, 13(1), 70-77.

Davis, A., Smith, P., Ferguson, M., Stephens, D., \& Gianopoulos, I. (2007). Acceptability, benefit and costs of early screening for hearing disability: A study of potential screening tests and models. Health Technology Assessment, 11(42), 1-294.

Henshaw, H. \& Ferguson, M. A. (2013). Efficacy of individual computerbased auditory training for people with hearing loss: A systematic review of the evidence. PLoS One, 8(5), e62836.

Hong, H. N. (2013). Case study of auditory training for the acquired hearing loss adult with cochlear implant. Journal of Rehabilitation Research, 17(4), 371-382.

Jang, H. S. (2007). Auditory training and auditory rehabilitation program. Rehabilitation Information, 20, 108-118.

Kim, H. G. \& Lee, K. W. (2010). Effects of word recognition score as a function of auditory training terms for elderly hearing impaired with hearing aid. Audiology and Speech Research, 6(2), 159-163.

Kim, J. \& Lee, K. (2017). Effects on word and sentence recognition by auditory training using environmental sound for elderly hearing impaired. Audiology and Speech Research, 13(2), 115-122.

Kim, S. \& Lee, K. (2018). Auditory training in an elderly hearing aid user using environmental sounds, stories and questions: A case report. $\mathrm{Au}$ diology and Speech Research, 14(3), 204-209.

Kim, Y. K. \& Lee, J. (2017). Effect of computer-based working memory training on word recognition and phonological processing for children with cochlear implants: A pilot study. The Journal of Humanities and Social Science, 8(4), 281-298. 
Kim, Y. M. \& Seok, D. I. (1998). The effect of language rehabilitation auditory comprehension program on auditory comprehension ability of hearing disordered students. Journal of Speech and Hearing Disorders, 7(2), 117-129.

Kochkin S. (2009). MarkeTrak VIII: 25 -year trends in the hearing health market. Hearing Review, 16(11), 12-31.

Kwon, S. H. (2005). The effects of self-language monitoring for improving functional language ability in children with cochlear implant. The Journal of Special Education, 44(2), 27-47.

Lee, J. H., Lee, S. G., \& Bahng, J. (2016). Case study of auditory training for long-term users of hearing aids with poor word recognition. Audiology and Speech Research, 12(3), 190-194.

Lee, M. S. \& Cho, S. J. (2011). A case report on aural rehabilitation after cochlear implantation in a child with LEOPARD syndrome. The Journal of Special Children Education, 13(3), 357-370.

Lee, M. S. \& Seo, Y. K. (2015). A case report on aural rehabilitation after cochlear implantation in a child with Goldenhar syndrome. The Journal of Special Children Education, 17(4), 77-93.

Lee, Y. \& Lee, S. (2017). Effect of smartphone applications for aural habilitation in cochlear implanted children: Improvement for auditory memory skills. Audiology and Speech Research, 13(2), 176-192.

Moher, D., Liberati, A., Tetzlaff, J., Altman, D. G.; PRISMA Group. (2009). Preferred reporting items for systematic reviews and meta-analyses: The PRISMA statement. PLoS Medicine, 6(7), e1000097.

Park, S. A., Shin, H. J., \& Park, E. S. (2019). The effect of nonsense two-syllable minimal contrast pair auditory training on school-aged cochlear implant wearers improvement in final plosive consonant auditory perception. Journal of Speech-Language and Hearing Disorders, 28(1), 5969.

Park, S. H. \& Lee, D. J. (2005). Aural rehabilitation of children with cochlear implants. Audiology and Speech Research, 1(1), 90-93.

Park, S. H., Seok, D. I., Jung. O. R., \& Lee, S. H. (2003). A study of the effects of multisensory auditory rehabilitation program on cochlear implanted children during off-switch state. Communication Sciences and Disorders, 8(3), 228-252.

Tye-Murray, N. (1998). Foundations of Aural Rehabilitation: Children, Adults, and Their Family Members. (1st ed.). Clifton Park, NY: Delmar Cengage Learning.

World Health Organization. (2019, March 20). Deafness and Hearing Loss. World Health Organization. Retrieved from https://www.who.int/newsroom/fact-sheets/detail/deafness-and-hearing-loss.

Yeo, S., Bahng, J., \& Lee, J. H. (2014). Efficacy of auditory training using sentences in noise for hearing aid users. Audiology and Speech Research, 10(1), 65-75.

Yoon, J., Kim, J., \& Park, H. (2016). Early auditory rehabilitation of an infant with unilateral profound hearing loss: A case study. Audiology and Speech Research, 12(2), 115-125.

Yu, J., Chun, H., Song, C. G., \& Han, W. (2014). Case study of speech perception enhancement in hearing-impaired adult by auditory training program of mobile device. Audiology and Speech Research, 10(2), 158168. 